\title{
Targeted IL-15-based Protein Fusion Complexes as Cancer Immuno- therapy Approaches
}

\author{
Sarah Alter ${ }^{1}$, Peter R. Rhode ${ }^{1}$, Emily K. Jeng ${ }^{1}$, and Hing C. Wong ${ }^{1 *}$ \\ 'Altor BioScience, Miramar, Florida
}

Article Info

\section{Article Notes}

Received: December 21, 2017

Accepted: January 16, 2018

\section{*Correspondence:}

Dr Hing C. Wong, Founder and Chief Executive Officer, Altor BioScience

2810 North Commerce Parkway

Miramar, FL 33025, USA,

E-mail: hingwong@altorbioscience.com

(C) 2018 Wong HC. This article is distributed under the terms of the Creative Commons Attribution 4.0 International License.

\section{Keywords:}

Interleukin-15

IL-15 superagonist

ALT-803

scaffold protein

fusion protein

immunotherapy

\section{ABSTRACT}

This mini review provides an overview and rationale for creating IL-15based fusion protein complexes to be used as targeted immunotherapeutic agents. IL-15 stimulates proliferation and activation of $\mathrm{CD}^{+} \mathrm{T}$ and natural killer cells which result in augmentation of their anti-tumor activities. We have created ALT-803, an IL-15 superagonist complex which exhibits longer serum half-life, longer retention in lymphoid tissues, and better immunostimulatory and anti-tumor activities compared to native IL-15. When used alone or in combination with other immunotherapeutic molecules in various mouse tumor models, ALT-803 effectively reduces tumor burden and prolongs survival by stimulating the innate and adaptive arms of the immune system. To evaluate whether ALT-803 could be used as a protein scaffold to create IL-15based tumor cell-specific molecules, we genetically fused it with a single chain anti-CD20 antibody derived from the variable regions of rituximab. This novel fusion protein exhibits enhanced anti-tumor activity compared to rituximab while maintaining IL-15 immunostimulating properties. Thus, ALT-803 may be exploited as a versatile scaffold to produce multivalent targeted fusion proteins to improve the anti-tumor efficacy of other therapeutic agents in the clinic.

\section{Interleukin-15 and ALT-803}

Common gamma chain $(\gamma c)$ cytokines, which include interleukin (IL)-2, IL-4, IL-7, IL-9, IL-15, and IL-21, have been studied extensively and shown to be promising as cancer immunotherapeutic agents ${ }^{1}$. Recombinant IL-2 (rIL-2) has been approved for treatment of patients with metastatic renal cell carcinoma and melanoma more than two decades ago. Although rIL-2 can induce durable and major responses in a subset of patients ${ }^{2,3}$, the use of rIL-2 at the effective dose level causes severe side effects, such as capillary leak syndrome and hypertension, requiring extensive in-patient care during its administration ${ }^{4}$. These treatment-induced side effects curtail the clinical utilities of rIL-2 as an immunotherapeutic drug.

IL-15, a four helix $\gamma c$ cytokine, is structurally related to rIL-2 ${ }^{5}$. The two $\gamma c$ cytokines use the same IL-2/IL-15 receptor $\beta \gamma c$ (IL$2 / 15 \mathrm{R} \beta \gamma \mathrm{c}$ ) displayed on the surface of natural killer (NK) and $\mathrm{T}$ cells for signaling ${ }^{1}$. However, IL-15 has been proven to stimulate anti-tumor immune responses of $\mathrm{NK}$ and $\mathrm{T}$ cells without the induction of IL-2-associated capillary leak syndrome, activationinduced cell death, and expansion of $\mathrm{T}$ regulatory cells ${ }^{1,6}$. Thus, IL-15 is considered a promising immunotherapeutic for cancer treatment ${ }^{3}$. IL-15 associates with IL-15 receptor $\alpha$ (IL-15R $\left.\alpha\right)$ on 
monocytes and dendritic cells and is trans-presented to form a complex with the IL-2/15R $\beta \gamma c$ on NK and T cells resulting in their activation ${ }^{5,7}$. To develop an IL-15-based therapeutic agent, we created a soluble complex, ALT803, consisting of two protein subunits of a human IL-15 variant with an asparagine to aspartic acid substitution at position 72 (N72D) associated to a dimeric human IL$15 R \alpha$ sushi (IL-15R $\alpha$ Su) domain/human IgG1 Fc fusion protein (IL-15R $\alpha \mathrm{SuFC}$ ) (Figure $1 \mathrm{~A})^{8,9}$. The N72D mutation increases the binding affinity of IL-15 to the IL-2/15R $\beta \gamma \mathrm{c}$ complex approximately 4- to 5-fold and significantly enhances the IL-15 biological activities ${ }^{8}$. Compared to native IL-15, ALT-803 exhibits increased in vivo half-life ( 25 hours versus $<40$ minutes for native IL-15) ${ }^{9}$ and better distribution to the lymphoid organs (i.e., lymph nodes, spleen) for prolonged immune cell stimulation ${ }^{10}$. ALT-803 is capable of simultaneously activating innate and adaptive immune cells and eliciting both rapid and long lasting antitumor activity as well as providing protective anti-tumor immunity $^{10-19}$. In pre-clinical models, systemic ALT-803 administration leads to antigen-independent proliferation, activation, and differentiation of memory $\mathrm{CD} 8^{+} \mathrm{CD} 44^{\text {high }} \mathrm{T}$ cells into potent effector cells and upregulation of NKG2D (KLRK1), but not CD25 or PD-1 ${ }^{10,11,13,14,17}$. These CD8 ${ }^{+}$T cells exhibit increased cytotoxic activity against tumor cells, as shown by secretion of high levels of IFN $\gamma$, granzyme $B$, and perforin $^{10,11}$. In addition to T cell stimulation, ALT-803 also induces $\mathrm{NK}$ cell proliferation, degranulation (measured by CD107a expression), IFN $\gamma$ and TNF $\alpha$ production, and tumor cell killing $10,12,13,15,17-19$. ALT-803 was also shown to promote robust expansion of NK cells with the highest fold increase observed in "high effector" $\mathrm{CD} 11 \mathrm{~b}^{+} \mathrm{CD} 27^{\text {high }}$ NK cells which are resistant to self-tolerance, are highly cytotoxic, and exhibit increased cytokine production and migratory capacity ${ }^{13}$. Felices et al. demonstrated that ALT803 can restore function of ovarian cancer patient ascitesderived NK cells and enhance cytotoxicity against ovarian tumor cells in vitro and in vivo ${ }^{18}$. In non-human primates, ALT-803 treatment results in increased peripheral blood $\mathrm{CD}^{+}, \mathrm{CD}^{+} \mathrm{T}$ and $\mathrm{NK}$ cell levels following four consecutive weekly doses with no significant treatment-dependent adverse effects ${ }^{10}$.

Another mechanism by which ALT-803 enhances NK cell-mediated lysis of tumor cells is through antibodydependent cellular cytotoxicity (ADCC) $)^{15,20}$. Short-term ALT-803 stimulation of human NK cells in vitro was shown to enhance rituximab-directed ADCC against Daudi and Raji tumor cell lines. ALT-803 combination treatment with rituximab in B-cell lymphoma-bearing mice results in significant reduction of tumor burden and increased survival when compared to treatment with rituximab alone ${ }^{15}$. Additionally, when combined with other potential immunotherapeutic molecules, such as checkpoint inhibitors, NKG2D ligand neutralizing antibody, or MEK inhibitor, ALT-803 improves the efficacy and durability of anti-tumor responses ${ }^{12-14,16,20}$. Combining the immunostimulatory property of ALT- 803 with the blockade of inhibitory immune checkpoints in multiple in vivo tumor models has been shown to increase the therapeutic effect of various checkpoint inhibitors (i.e., PD-1, PD-L1, and CTLA-4) by promoting development and function of effector $\mathrm{NK}$ and $\mathrm{CD}^{+} \mathrm{T}$ cells and mediating a more potent anti-tumor activity versus checkpoint inhibition alone ${ }^{13,14}$. Therefore, although ALT-803 exhibits anti-tumor efficacy as a monotherapy, it also has great potential for use in combination with other immunotherapeutic agents. Importantly, mice cured by ALT-803 treatment have been shown to be resistant to tumor rechallenge, which indicates that ALT-803 also induces long-term memory and durable anti-tumor immunity ${ }^{11,14,16}$. As a result, ALT-803 is being developed as a potential cancer immunotherapeutic and is currently in multiple clinical trials for treatment of patients with advanced solid and hematologic malignancies.

\section{ALT-803-based Protein Scaffold for Targeted Immunotherapeutics}

ALT-803 may act as a versatile protein scaffold, which can be modified for the creation of novel target-specific immunotherapeutic agents ${ }^{8,21,22}$. We previously showed that IL-15 binding and function are preserved when ALT803 components are fused to single chain T cell receptor (TCR) antigen binding domains ${ }^{21}$. These targeted fusion proteins consist of TCRs recognizing a peptide of human p53 protein (c264scTCR or c149scTCR), or an OVA protein epitope (OT1scTCR), linked to either IL-15, IL-15N72D, or IL-15R $\alpha$ Su N termini ${ }^{8,21}$. Dimerization through IL-15:IL$15 \mathrm{R} \alpha$ or IL-15N72D:IL-15R $\alpha$ interaction was shown to maintain the individual TCR's functional activity and increase effective antigen binding through enhanced avidity $^{21}$. Additionally, TCR fusion to $\mathrm{N}$ termini of either IL-15:IL-15R $\alpha \mathrm{Su}$ or IL-15N72D:IL-15R $\alpha$ Su scaffold provides the spatial orientation necessary for functionally independent heterodimer complexes while retaining flexibility to allow folding of closely paired TCR chains ${ }^{21}$. These fusion proteins are readily produced and easily purified from cell culture supernatant at a relatively high level. Since ALT-803 is comprised of an IL-15N72D:IL$15 \mathrm{R} \alpha \mathrm{SuFc}$ dimer, the results of the studies discussed above suggest that ALT-803 can be used to generate multivalent disease-specific fusion proteins (Figure 1).

Based on previous data demonstrating that ALT-803 can enhance rituximab's anti-tumor activity, we created 2B8T2M, a novel CD20-specific fusion protein complex. Four single chain domains of the monoclonal antibody, rituximab, were genetically fused to $\mathrm{N}$ termini of IL-15N72D and IL-15R $\alpha$ SuFc proteins ${ }^{22}$. This molecule exhibits trispecific binding activity through its recognition of CD20 on B lymphoma cells, IL-2/15R $\beta \gamma c$ on immune cells, and 


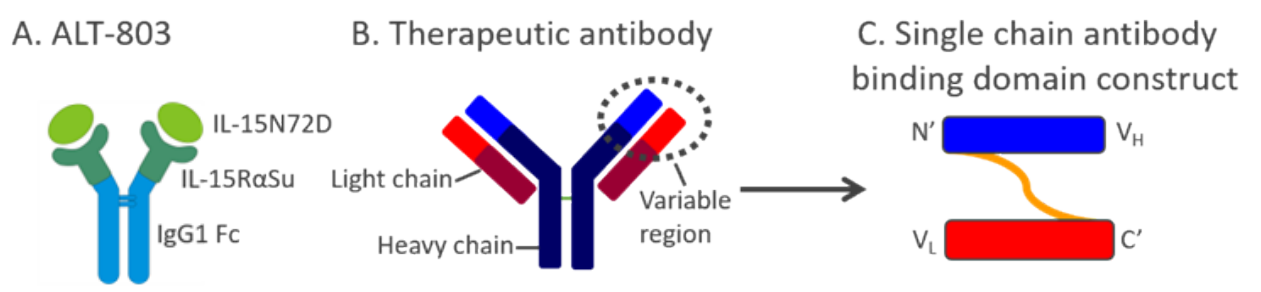

D. Fusion protein constructs

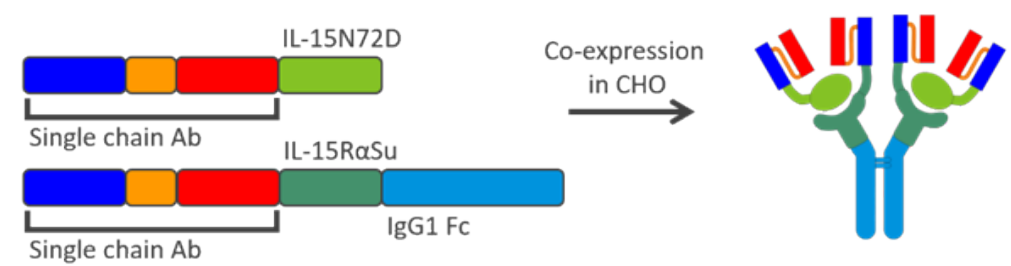

Figure 1. ALT-803 as a scaffold for creation of novel fusion molecules

A schematic diagram showing construction of novel fusion molecules using ALT-803 as a scaffold and a therapeutic antibody as an example. A. ALT-803 structure:IL-15N72D:IL-15R $\alpha$ SuFc complex consisting of IL-15N72D associated with the dimeric IL-15R $\alpha$ SuFc fusion protein. B. Structure of a therapeutic antibody showing the variable region consisting of the light and heavy chains. C. Variable gene segment of the antibody light chain linked to the $5^{\prime}$ end of the variable gene segment of the antibody heavy chain via a Gly ${ }_{4} \operatorname{Ser}_{3}$ linker to create a single chain antibody. D. A single chain antibody construct genetically fused to the $5^{\prime}$ end of the IL-15N72D mutein and a single chain antibody construct genetically fused to the $5^{\prime}$ end of the IL-15R $\alpha$ SuFc construct. E. A soluble novel ALT-803-based fusion molecule is produced. The fusion protein is comprised of four single chain antibody domains: two are fused to the two IL-15N72D muteins and two are fused to the two IL-15R $\alpha$ SuFc fusion constructs.

Fc receptor on NK cells and macrophages (Figure 2). When cultured with Daudi tumor cells, 2B8T2M shows enhanced pro-apoptotic activity that is comparable to $>600$-fold higher concentration of rituximab alone and is dependent on CD20 binding. Additionally, 2B8T2M is significantly more effective at inducing ADCC against Daudi tumor cells by human immune cells compared to rituximab ${ }^{22}$. A biodistribution study of the fusion protein demonstrated that adding a targeting domain to ALT-803 does not alter the molecule's ability to home to lymphoid tissues and be retained there for at least 70 hours $^{22}$. In Daudi lymphomabearing mice, 2B8T2M treatment results in significantly lower tumor burden and improved survival compared to rituximab treatment alone. Specific B cell depletion by 2B8T2M was also observed in cynomolgus monkeys and importantly, no significant adverse events were observed ${ }^{22}$. These results demonstrate that fusing a single chain antibody domain to ALT-803 is an effective strategy to potentiate tumor targeting immunotherapeutic molecule without compromising its immunostimulatory capabilities. Based on these results, 2B8T2M is currently in late-stage pre-clinical development.

Generating a single agent with multiple tumor targets and anti-tumor activities may be more beneficial compared to a combination of individual molecules for treatment of cancer. Our data show that linking a CD20 targeting antibody to ALT-803 retains its superior IL-15 activity while mediating more specific tumor killing ${ }^{22}$. In addition to improved anti-tumor efficacy, the ALT-803 scaffold may

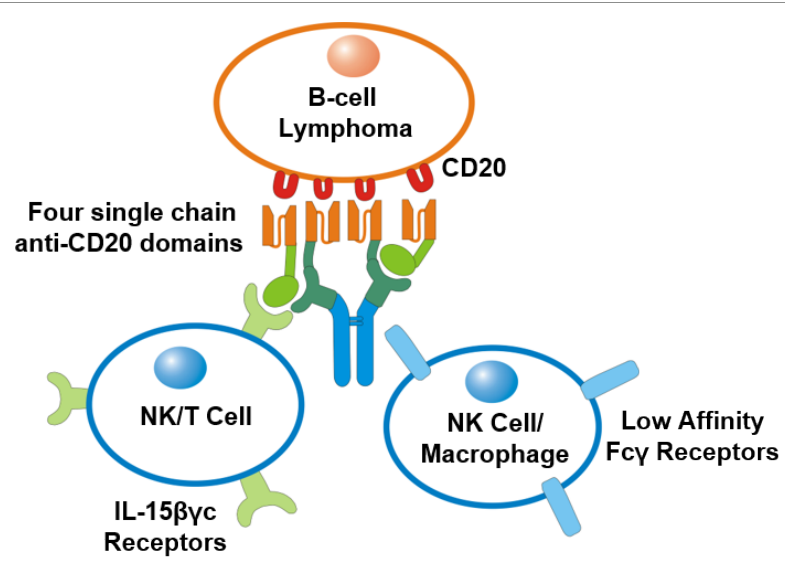

Figure 2. 2B8T2M exhibits tri-specific binding activity

2B8T2M, a fusion protein with four single chain anti-CD20 Ab domains fused to ALT-803, is shown to recognize and specifically bind the CD20 molecule on a B-cell lymphoma cell, as well as low affinity Fcy receptors on an NK cell or macrophage. Also shown is binding of the IL-15N72D:IL-15RaSu portion of 2B8T2M to IL$2 / 15 R \beta \gamma c$ on NK and T cells for immune stimulation.

also provide a vehicle for preferential delivery to lymphoid organs to allow proper and prolonged immune activation ${ }^{22}$.

\section{Future Opportunities}

In order to overcome the various immunological barriers and improve clinical outcomes in cancer, immunotherapeutics must focus on stimulating different cellular immune pathways and overcoming the highly suppressive tumor microenvironment. IL-15-mediated 
immune cell activation by ALT-803 is expected to be attenuated by induction of immune checkpoint such as PD-1 expression by immune effector cells and PD-L1 expression by tumor cells ${ }^{23,24}$. Co-administration of checkpoint inhibitors, such as anti-PD-1 and anti-PD-L1, with ALT803 may aid in overcoming the immunosuppressive tumor microenvironment and prolonging ALT-803-stimulated $\mathrm{T}$ and NK cell activity and persistence. Potent immune stimulation and blocking suppression can be achieved in a single therapeutic agent using the ALT-803 protein scaffold by combining the highly potent immunostimulatory cytokine, IL-15, with checkpoint inhibitors for preventing immune exhaustion, therapeutic monoclonal antibodies and TCRs for disease-cell targeting, and protein-based molecules for neutralizing immune suppression. Also, ALT803 could be used to create a single molecule fused with various cytokines for exogenous expansion and stimulation of immune cells for adoptive cell therapy. Adding infectious agent-specific recognition domains to the ALT-803 scaffold has the potential to create novel fusion proteins against virally infected cells for treatment of infectious diseases as well. Thus, ALT-803 is versatile protein scaffold that provides an opportunity for creating unlimited numbers of novel and highly potent immunostimulatory fusion molecules for various clinical applications.

\section{Conflict of interest statement}

The authors are employees and shareholders of Altor BioScience.

\section{References}

1. Waldmann TA. The biology of interleukin-2 and interleukin-15: implications for cancer therapy and vaccine design. Nat Rev Immunol. 2006; 6(8): 595-601.

2. Atkins MB, Lotze MT, Dutcher JP, et al. High-dose recombinant interleukin 2 therapy for patients with metastatic melanoma: analysis of 270 patients treated between 1985 and 1993. J Clin Oncol. 1999; 17(7): 2105-2116.

3. Cheever MA. Twelve immunotherapy drugs that could cure cancers. Immunol Rev. 2008; 222: 357-368.

4. Rosenberg SA. Interleukin-2 and the development of immunotherapy for the treatment of patients with cancer. Cancer J Sci Am. 2000; 6 Suppl 1: S2-7.

5. Waldmann T, Tagaya Y, Bamford R. Interleukin-2, interleukin-15, and their receptors. Int Rev Immunol. 1998; 16(3-4): 205-226.

6. Waldmann TA, Lugli E, Roederer M, et al. Safety (toxicity), pharmacokinetics, immunogenicity, and impact on elements of the normal immune system of recombinant human IL-15 in rhesus macaques. Blood. 2011; 117(18): 4787-4795.

7. Dubois S, Mariner J, Waldmann TA, et al. IL-15Ralpha recycles and presents IL-15 In trans to neighboring cells. Immunity. 2002; 17(5): 537-547.
8. Zhu X, Marcus WD, Xu W, et al. Novel human interleukin-15 agonists. J Immunol. 2009; 183(6): 3598-3607.

9. Han KP, Zhu X, Liu B, et al. IL-15:IL-15 receptor alpha superagonist complex: high-level co-expression in recombinant mammalian cells, purification and characterization. Cytokine. 2011; 56(3): 804-810.

10. Rhode PR, Egan JO, Xu W, et al. Comparison of the Superagonist Complex, ALT-803, to IL15 as Cancer Immunotherapeutics in Animal Models. Cancer Immunol Res. 2016; 4(1): 49-60.

11. Xu W, Jones M, Liu B, et al. Efficacy and mechanism-of-action of a novel superagonist interleukin-15: interleukin-15 receptor alphaSu/ Fc fusion complex in syngeneic murine models of multiple myeloma. Cancer Res. 2013; 73(10): 3075-3086.

12. Basher F, Jeng EK, Wong $\mathrm{H}$, et al. Cooperative therapeutic anti-tumor effect of IL-15 agonist ALT-803 and co-targeting soluble NKG2D ligand sMIC. Oncotarget. 2016; 7(1): 814-830.

13. Kim PS, Kwilas AR, Xu W, et al. IL-15 superagonist/IL-15RalphaSushiFc fusion complex (IL-15SA/IL-15RalphaSu-Fc; ALT-803) markedly enhances specific subpopulations of NK and memory CD8+ T cells, and mediates potent anti-tumor activity against murine breast and colon carcinomas. Oncotarget. 2016; 7(13): 16130-16145.

14. Mathios D, Park CK, Marcus WD, et al. Therapeutic administration of IL-15 superagonist complex ALT-803 leads to long-term survival and durable antitumor immune response in a murine glioblastoma model. Int J Cancer. 2016; 138(1): 187-194.

15. Rosario M, Liu B, Kong L, et al. The IL-15-Based ALT-803 Complex Enhances FcgammaRIIIa-Triggered NK Cell Responses and In Vivo Clearance of B Cell Lymphomas. Clin Cancer Res. 2016; 22(3): 596-608.

16. Allegrezza MJ, Rutkowski MR, Stephen TL, et al. IL15 Agonists Overcome the Immunosuppressive Effects of MEK Inhibitors. Cancer Res. 2016; 76(9): 2561-2572.

17. Bailey CP, Budak-Alpdogan T, Sauter CT, et al. New interleukin-15 superagonist (IL-15SA) significantly enhances graft-versus-tumor activity. Oncotarget. 2017; 8(27): 44366-44378.

18. Felices M, Chu S, Kodal B, et al. IL-15 super-agonist (ALT-803) enhances natural killer (NK) cell function against ovarian cancer. Gynecol Oncol. 2017; 145(3): 453-461.

19. Wagner JA, Rosario M, Romee R, et al. CD56bright NK cells exhibit potent antitumor responses following IL-15 priming. J Clin Invest. 2017; 127(11): 4042-4058.

20. Jochems C, Tritsch SR, Pellom ST, et al. Analyses of functions of an antiPD-L1/TGFbetaR2 bispecific fusion protein (M7824). Oncotarget. 2017; 8(43): 75217-75231.

21. Wong RL, Liu B, Zhu X, et al. Interleukin-15:Interleukin-15 receptor alpha scaffold for creation of multivalent targeted immune molecules. Protein Eng Des Sel. 2011; 24(4): 373-383.

22. Liu B, Kong L, Han K, et al. A Novel Fusion of ALT-803 (Interleukin (IL)15 Superagonist) with an Antibody Demonstrates Antigen-specific Antitumor Responses. J Biol Chem. 2016; 291(46): 23869-23881.

23. Yu P, Steel JC, Zhang M, et al. Simultaneous blockade of multiple immune system inhibitory checkpoints enhances antitumor activity mediated by interleukin-15 in a murine metastatic colon carcinoma model. Clin Cancer Res. 2010; 16(24): 6019-6028.

24. Yu P, Steel JC, Zhang M, et al. Simultaneous inhibition of two regulatory T-cell subsets enhanced Interleukin-15 efficacy in a prostate tumor model. Proc Natl Acad Sci U S A. 2012; 109(16): 6187-6192. 